\title{
Pellet Dosage Form Category
}

National Cancer Institute

\section{Source}

National Cancer Institute. Pellet Dosage Form Category. NCI Thesaurus. Code C154590.

A type of solid pharmaceutical dose form consisting of usually cylindrical masses, usually formed by extrusion. 